\title{
Early hemodynamic assessment using NICOM in patients at risk of developing Sepsis immediately after emergency department triage
}

Steve B. Chukwulebe ${ }^{1}$, David F. Gaieski ${ }^{2 *}$, Abhishek Bhardwaj ${ }^{3}$, Lakeisha Mulugeta-Gordon ${ }^{4}$, Frances S. Shofer ${ }^{5}$ and Anthony J. Dean ${ }^{5}$

\begin{abstract}
Background: One factor leading to the high mortality rate seen in sepsis is the subtle, dynamic nature of the disease, which can lead to delayed detection and under-resuscitation. This study investigated whether serial hemodynamic parameters obtained from a non-invasive cardiac output monitor (NICOM) predicts disease severity in patients at risk for sepsis.

Methods: Prospective clinical trial of the NICOM device in a convenience sample of adult ED patients at risk for sepsis who did not have obvious organ dysfunction at the time of triage. Hemodynamic data were collected immediately following triage and 2 hours after initial measurement and compared in two outcome groupings: (1) admitted vs. dehydrated, febrile, hypovolemicdischarged patients; (2) infectious vs. non-infectious sources. Receiver operator characteristic (ROC) curves were calculated to determine whether the NICOM values predict hospital admission better than a serum lactate.
\end{abstract}

Results: 50 patients were enrolled, 32 (64\%) were admitted to the hospital. Mean age was $49.5( \pm 16.5)$ years and $62 \%$ were female. There were no significant associations between changes in hemodynamic variables and patient disposition from the ED or diagnosis of infection. Lactate was significantly higher in admitted patients and those with infection ( $p=0.01, p=0.01$ respectively). The area under the ROC [95\% Confidence Intervals] for lactate was 0.83 [0.64-0.92] compared to 0.59 [0.41-0.73] for cardiac output (CO), 0.68 [0.49-0.80] for cardiac index (CI), and 0.63 [0.36-0.80] for heart rate (HR) for predicting hospital admission.

Conclusions: $\mathrm{CO}$ and $\mathrm{Cl}$, obtained at two separate time points, do not help with early disease severity differentiation of patients at risk for severe sepsis. Although mean HR was higher in those patients who were admitted, a serum lactate still served as a better predictor of patient admission from the ED.

Keywords: Severe sepsis, Noninvasive monitoring, Cardiac output, Cardiac index, Heart rate, Lactate

\footnotetext{
* Correspondence: david.gaieski@jefferson.edu

${ }^{2}$ Department of Emergency Medicine, Sidney Kimmel Medical College at

Thomas Jefferson University, 1025 Walnut Street; 300 College Building, 19107 Philadelphia, PA, USA

Full list of author information is available at the end of the article
}

(C) The Author(s). 2021 Open Access This article is licensed under a Creative Commons Attribution 4.0 International License, which permits use, sharing, adaptation, distribution and reproduction in any medium or format, as long as you give appropriate credit to the original author(s) and the source, provide a link to the Creative Commons licence, and indicate if changes were made. The images or other third party material in this article are included in the article's Creative Commons licence, unless indicated otherwise in a credit line to the material. If material is not included in the article's Creative Commons licence and your intended use is not permitted by statutory regulation or exceeds the permitted use, you will need to obtain permission directly from the copyright holder. To view a copy of this licence, visit http://creativecommons.org/licenses/by/4.0/ The Creative Commons Public Domain Dedication waiver (http://creativecommons.org/publicdomain/zero/1.0/) applies to the data made available in this article, unless otherwise stated in a credit line to the data. 


\section{Background}

Sepsis, defined as a dysregulated inflammatory response syndrome to an infectious trigger, causing acute organ dysfunction, is one of the leading causes of death worldwide [1-3]. The mortality is greater than $15 \%$ for sepsis and greater than $20 \%$ for septic shock [2]. One factor leading to the high mortality rate seen in sepsis is the subtle, dynamic nature of the disease, which can lead to delayed detection and under-resuscitation. Because of the natural progression of sepsis, patients often present to the Emergency Department (ED) dehydrated, febrile, hypovolemic, hyperdynamic, and vasoconstricted. Initial pro-inflammatory effects act to increase capillary permeability causing fluid shifts into the interstitial space, resulting in further hypovolemia, and, in a subset of patients, depressed myocardial function. Varying hemodynamic profiles can be present during the proximal phase of sepsis in the ED [4]. Later, antiinflammatory stages of the syndrome can cause immunomodulatory dysfunction and multiorgan failure [3]. Since the treatment of hypovolemia and myocardial depression involves different and at times competing modalities, an understanding of a patient's specific hemodynamic profile early in the treatment course may improve outcomes.

Numerous studies have examined various biomarkers that track the body's response to bacterial infection, systemic inflammation, tissue perfusion, and systemic oxygen delivery, and have identified them as significant predictors of severity of illness [5-7]. The most promising of these biomarkers is lactate and elevated lactate levels are associated with myocardial dysfunction, hypoperfusion, and mortality $[8,9]$. However, a patient with a significantly elevated lactate level can have supranormal, normal, or depressed hemodynamic function $[8,9]$. Thus, it has been suggested that other non-invasive means of prognosticating patients at risk for severe sepsis-associated hemodynamic deterioration are needed. To date, few studies have examined non-invasive means of tracking the hemodynamic changes in sepsis.

While pulmonary artery catheterization (PAC) historically has been the most studied method of monitoring hemodynamics, there are risks associated with PAC placement and limitations to the information obtained. A recent literature review regarding the use of PAC in sepsis patients by Karanikolas et al. found that there is no significant benefit in regards to outcomes [10]. Other techniques such as a transthoracic echocardiography, thoracic bioimpedance, exhaled $\mathrm{CO}_{2}$ measured by Fick's principle, and arterial pulse contour have been assessed with variable agreement to PAC measurement [3, 1113]. The Noninvasive Cardiac Output Monitor (NICOM; Cheetah Medical) uses bioreactance to determine cardiac output $(\mathrm{CO})$ [14]. Bioreactance $(\mathrm{d} \Phi)$ is measured by a change in the phase of a voltage signal sent from an electrode on one side of the chest to another electrode on the contralateral side. Peak rate of change is proportional to peak aortic flow. Thus, stroke volume (SV) can be calculated by the following formula, SV $=\mathrm{C} \mathrm{x}$ VET $\mathrm{x}$ $(\mathrm{d} \Phi / \mathrm{dt})_{\max }$. $\mathrm{C}$ is a constant defined by the manufacturer and VET is the ventricular ejection time determined from the NICOM device by analysis of the electrocardiographic signals. Once SV is determined, that value can be used to compute $\mathrm{CO}$. Cardiac Index (CI) can also be calculated if the patient's height and weight are obtained. Although the NICOM has been studied in perioperative settings and intensive care units (ICU), relatively few studies have looked at its use in the non-traumatic ED patient [15-21]. Furthermore, there are no studies that use the NICOM as a way to prognosticate which patients presenting to the ED without obvious signs of acute organ injury are at greatest risk for developing severe sepsis.

This is a pilot study of NICOM to determine whether the hemodynamic parameters it provides can be used in triage or in a serial fashion to predict severity of disease in stable-appearing patients at risk for sepsis on ED presentation. Initial triage NICOM values obtained as soon as possible after ED triage as well as their change at a second time point two hours later were used to test the following three hypotheses: (1) NICOM values recorded over the first two hours in the ED, while stable patients often remain in the waiting room prior to initiation of treatment, will predict admission to the hospital when comparing admitted vs. discharged patients; (2) NICOM values recorded over the first two hours in the ED will differ in patients with an infectious vs. noninfection source; and (3) given the association of lactate with organ dysfunction and mortality in sepsis, NICOM values recorded over the first two hours in the ED will correlate with serum lactate levels in their efficacy to predict patient disposition from the ED.

\section{Methods \\ Study Design and setting}

This study was a prospective, observational, convenience sample study of the NICOM during a 4-month enrollment period from October 27th, 2014 to February 23rd, 2015.

\section{Population}

The study was performed in the ED at an urban quaternary care center in a large metropolitan area.

Patients presenting to the ED and triaged as an Emergency Severity Index (ESI) 2 or 3, suggesting they can wait between 20 minutes and 2 hours to begin treatment, were eligible for enrollment if they met the following inclusion criteria: 
1. Age $\geq 18$ years;

2. At least 2 of the 3 SIRS criteria measured at triage (Temperature $<96.8^{\circ} \mathrm{F}$ or $>100.4^{\circ} \mathrm{F}$; Heart rate $(\mathrm{HR})>90$ beats per minute (BPM); Respiratory rate $(\mathrm{RR})>20$ breaths/minute) were present;

3. An automatic lactate order was generated by the ED Advanced Triage Protocol (ATP), which required an ESI classification of ESI 2 or 3, the presence of at least 2 SIRS criteria in triage, AND a chief complaint consistent with infection. The presence of at least two triage SIRS criteria and one of the 50 pre-identified chief complaints that suggest they may have a focus of infection automatically prompts an order for a serum lactate through the HUP ED ATP [Appendix]. The goal of the ATP process is to expedite a serum lactate result to help risk stratify patients with possible sepsis in a patient population that includes patients who have the potential to decompensate during the period when they are waiting for treatment to begin.

Patients were excluded from the study if any of the following were present:

1. Assigned ESI 1, requiring immediate treatment by the clinical team;

2. Assigned either ESI 4 or 5, categorizing them as "stable to wait" for several hours for treatment to begin and assigned to be seen in the Fast Track section of the ED;

3. Altered mental status;

4. Inability to obtain informed consent;

5. Non-English speaking.

Patients who met inclusion criterion 3, the ED ATP, generated a text message to the research team and their records were reviewed. Patients who met all inclusion and exclusion criteria during a time when study personnel were available were approached for enrollment in the study and informed consent was obtained. Patient enrollment, chart review, and data collection was performed by a single observer (SBC). The study protocol was approved by the University of Pennsylvania's Institutional Review Board prior to beginning patient enrollment.

\section{Demographics, historical, Triage, and Laboratory Data}

Patient demographics including age, sex, race, weight, and height were collected. Triage data collected included temperature, HR, blood pressure, and RR. Comorbidities collected included a history of diabetes, coronary artery disease, congestive heart failure, chronic obstructive pulmonary disease, cancer, human immunodeficiency virus status, organ transplantion, systemic lupus erythematosus, smoking, and surgery in the month prior to triage presentation. Laboratory values collected include serum lactate, complete blood count, and chemistry panels.

\section{NICOM Data Collection}

After informed consent was obtained, coincident with obtaining of a blood specimen to assess triage serum lactate level, enrolled patients were placed in a semirecumbent position with the head of the bed at $45^{\circ}$ and their feet fully extended. The NICOM sensors and blood pressure cuff were applied to the patient according to the manufacturer's specifications. The device was allowed to calibrate for 3-5 minutes, at which point single values for the following data were collected and automatically stored: time, CO, CI, HR, blood pressure, mean arterial pressure (MAP), total peripheral resistance, total peripheral resistance index, SV, and stroke volume index (SVI). The pads were removed after the first measurements were obtained. After two hours, the same pads were replaced on the patient in approximately the same locations, and the process was repeated to obtain a second set of NICOM values. The values collected from the NICOM device were not available to the treating clinicians and were not used as part of patient management.

\section{Clinical outcomes}

In the primary outcome, NICOM values obtained at time zero and two hours were compared in patients who were admitted to the hospital vs. those who were discharged from the ED. NICOM values were also compared in patients who had an infectious source vs. those who did not have an infectious source. Patients with a diagnosis of infection were defined by examining the charts of all patients enrolled in the study for ED physician documentation that suggested suspected infection, an order for blood cultures, use of antibiotics in the ED, and need for hospital admission. Patient classification was conducted without knowledge of the NICOM values obtained at time zero and two hours later.

\section{Statistical analysis}

An analysis of variance in repeated measures (RMANOVA) was used to compare differences over time in the NICOM variables of $\mathrm{CO}, \mathrm{CI}, \mathrm{SV}$, and $\mathrm{HR}$ in each of the two outcome groupings (admitted vs. discharged; infectious source vs. non-infectious source). The NICOM HR was included in the analysis to determine if the post-triage measurement of the NICOM specific values of $\mathrm{CO}, \mathrm{CI}$, and SV provide risk stratification information when compared to the triage HR. Additionally, receiver operator characteristic (ROC) curves were created to compare the area under the curve (AUC) and determine 
whether the initial NICOM CO, CI, SV, and HR values or the change in these measurements at the second time point predict hospital admission better than a serum lactate. SAS (Version 9.4, SAS Institute, Cary, NC), NCSS (Version 8.0, NCSS LLC, Kaysville, UT), and SPSS (Version 17.0, International Business Machines Corp., Armonk, NY) software were used to perform the statistical analysis. $p<0.25$ was considered to be significant for interactions in the RM-ANOVA. A 2-sided p-value of $p<0.05$ was considered to be significant in other analyses.

\section{Results}

Approximately 100 text messages for patients meeting the ATP criteria were sent to study personnel during times when one was available to enroll potential patients and the potential patients' ED records were reviewed. Fifty patients were consented and enrolled during the study period; the mean age was $49.5( \pm 16.5)$ years; $62 \%$ were female; and $56 \%$ were African American. Cancer $(14 / 50 ; 28 \%)$ was the most common comorbidity, followed by Diabetes Mellitus (10/50; $20 \%)$. Of the 50 patients, $32(64 \%)$ were admitted to the hospital from the ED; 14 (28\%) were diagnosed as SIRS without evidence of infection, $24(48 \%)$ as infection or sepsis, 11 $(22 \%)$ as severe sepsis and $1(2 \%)$ patient as having septic shock. A higher percentage of septic vs. non-septic patients were admitted to the hospital. Patients admitted from the ED were significantly older ( 55.2 vs. 39.5 years old, $p=0.001$ ); more likely to be male ( $50 \%$ vs. $17 \%$, $p=0.02)$; and more likely to have a history of cancer ( $38 \%$ vs. $11 \%, P=0.05$ ) when compared to those who were discharged (Table 1). Patients with an infectious source were significantly older that those without an infectious source $(52.0$ vs. 42.8 years old, $\mathrm{P}=0.02)$. ATP lactate orders were generated on $100 \%$ (50/50) patients; $74 \%(37 / 50)$ had a lactate value drawn immediately after the order was generated; the mean lactate for this group was $1.5 \pm 0.8 \mathrm{mmol} / \mathrm{L}$.

Admitted patients had a higher triage RR ( 22 vs. 19 breaths per minute, $\mathrm{P}=0.01)$ and serum lactate $(1.7 \pm 0.8$ vs. $0.9 \pm 0.3 \mathrm{mmol} / \mathrm{L}, P=0.01)$ when compared to discharged patients (Table 2). Patients with an infectious source had a significantly higher lactate than those without an infectious source $(1.7 \pm 0.9$ vs. $1.0 \pm 0.4 \mathrm{mmol} / \mathrm{L}$, $P=0.01)$.

The average time from ED triage to 1st NICOM values was $38.0 \pm 17.4$ minutes and the range was $17-120 \mathrm{mi}-$ nutes. No patients received fluid resuscitation between the time zero and time 2-hour NICOM measurements. The mean values for $\mathrm{CO}, \mathrm{CI}$ and $\mathrm{HR}$ at time zero were $7.60 \mathrm{~L} / \mathrm{min}, 3.93 \mathrm{~L} / \mathrm{min} / \mathrm{m}^{2}$ and $109 \mathrm{BPM}$ and at time two hours were $6.88 \mathrm{~L} / \mathrm{min}, 3.58 \mathrm{~L} / \mathrm{min} / \mathrm{m}^{2}$ and $98 \mathrm{BPM}$ respectively. In the RM-ANOVA, there were no

Table 1 Patient characteristics

\begin{tabular}{|c|c|c|c|c|c|c|c|c|c|c|c|c|}
\hline \multirow{3}{*}{$\begin{array}{l}\text { Number (\% of enrolled) } \\
\text { Age, y }\end{array}$} & \multicolumn{2}{|c|}{ All Patients } & \multicolumn{2}{|c|}{ Admitted } & \multicolumn{2}{|c|}{ Discharged } & \multirow[t]{2}{*}{$P$ Value } & \multicolumn{2}{|c|}{ Infectious Source } & \multicolumn{2}{|c|}{ Non-Infectious Source } & \multirow[t]{2}{*}{$P$ Value } \\
\hline & \multicolumn{2}{|c|}{$N=50$} & \multicolumn{2}{|c|}{$N=32(64)$} & \multicolumn{2}{|c|}{$N=18(36)$} & & \multicolumn{2}{|c|}{$N=30(60)$} & \multicolumn{2}{|c|}{$N=20(40)$} & \\
\hline & 49.5 & \pm 16.2 & 55.2 & \pm 13.1 & 39.5 & \pm 16.7 & 0.001 & 54.0 & \pm 14.0 & 42.8 & \pm 17.3 & 0.015 \\
\hline Female sex, (\%) & 31 & $(62)$ & 16 & $(50)$ & 15 & $(83)$ & 0.020 & 17 & $(57)$ & 14 & $(70)$ & 0.341 \\
\hline African American, (\%) & 28 & (56) & 17 & (53) & 11 & $(61)$ & 0.426 & 18 & $(60)$ & 10 & $(50)$ & 0.459 \\
\hline Caucasian, (\%) & 20 & $(40)$ & 14 & $(44)$ & 6 & (33) & 0.426 & 11 & $(37)$ & 9 & $(45)$ & 0.459 \\
\hline Asian, (\%) & 1 & (2) & 0 & (0) & 1 & (6) & 0.426 & 0 & $(0)$ & 1 & (5) & 0.459 \\
\hline Weight (kg) & 84.8 & \pm 26.3 & 89.4 & \pm 27.7 & 76.8 & \pm 22.1 & 0.105 & 87.5 & \pm 25.7 & 80.9 & \pm 27.4 & 0.393 \\
\hline Height (in) & 65.7 & \pm 4.4 & 66.3 & \pm 4.7 & 64.7 & \pm 3.6 & 0.195 & 66.3 & \pm 4.7 & 65.0 & \pm 3.7 & 0.300 \\
\hline \multicolumn{13}{|l|}{ Patient History } \\
\hline CAD, (\%) & 1 & (2) & 1 & (3) & 0 & (0) & 0.449 & 0 & $(0)$ & 1 & (5) & 0.216 \\
\hline Cancer, (\%) & 14 & (28) & 12 & (38) & 2 & $(11)$ & 0.046 & 11 & (37) & 3 & $(15)$ & 0.095 \\
\hline $\mathrm{CHF},(\%)$ & 2 & (4) & 1 & (3) & 1 & (6) & 0.674 & 1 & (3) & 1 & (5) & 0.768 \\
\hline COPD, (\%) & 8 & (16) & 7 & $(22)$ & 1 & (6) & 0.131 & 6 & $(20)$ & 2 & (10) & 0.345 \\
\hline Diabetes mellitus, (\%) & 10 & $(20)$ & 7 & $(22)$ & 3 & $(17)$ & 0.659 & 6 & $(20)$ & 4 & $(20)$ & 1.000 \\
\hline $\mathrm{HIV},(\%)$ & 3 & (6) & 3 & (9) & 0 & $(0)$ & 0.180 & 3 & $(10)$ & 0 & (0) & 0.145 \\
\hline Lupus, (\%) & 1 & $(2)$ & 1 & (3) & 0 & (0) & 0.449 & 1 & (3) & 0 & $(0)$ & 0.409 \\
\hline Smoking history, (\%) & 3 & (6) & 2 & (6) & 1 & (6) & 0.921 & 2 & $(7)$ & 1 & (5) & 0.808 \\
\hline Surgery in past month, (\%) & 3 & (6) & 3 & (9) & 0 & (0) & 0.180 & 3 & (10) & 0 & (0) & 0.145 \\
\hline Transplant, (\%) & 4 & (8) & 3 & (9) & 1 & (6) & 0.633 & 2 & (7) & 2 & (10) & 0.670 \\
\hline
\end{tabular}

Data are presented as mean \pm standard deviation, or count (percentage). CAD indicates, coronary artery disease; CHF congestive heart failure; COPD chronic obstructive pulmonary disease; HIV human immunodeficiency virus 
Table 2 Triage, Laboratory Data and Outcomes

\begin{tabular}{|c|c|c|c|c|c|c|c|c|c|c|c|c|}
\hline \multirow{2}{*}{ Number (\% of enrolled) } & \multicolumn{2}{|c|}{ All Patients } & \multicolumn{2}{|c|}{ Admitted } & \multicolumn{2}{|c|}{ Discharged } & $P$ Value & \multicolumn{2}{|c|}{ Infectious Source } & \multicolumn{2}{|c|}{ Non-Infectious Source } & \multirow[t]{2}{*}{$P$ Value } \\
\hline & $N=50$ & & $N=32$ & (64) & $N=1 \varepsilon$ & (36) & & $N=30$ & & $N=20$ & & \\
\hline \multicolumn{13}{|l|}{ Initial Triage Vitals } \\
\hline Temperature, F & 100.4 & \pm 1.9 & 100.1 & \pm 1.9 & 100.9 & \pm 1.9 & 0.147 & 100.3 & \pm 1.9 & 100.5 & \pm 2.0 & 0.646 \\
\hline Heart rate, per min & 118.5 & \pm 15.4 & 120.9 & \pm 15.3 & 114.2 & \pm 15.0 & 0.140 & 120.5 & \pm 16.0 & 115.4 & \pm 14.2 & 0.254 \\
\hline $\mathrm{SBP}, \mathrm{mmHg}$ & 135.0 & \pm 25.1 & 136.9 & \pm 27.9 & 131.6 & \pm 19.3 & 0.472 & 132.4 & \pm 27.6 & 138.9 & \pm 20.8 & 0.375 \\
\hline $\mathrm{DBP}, \mathrm{mmHg}$ & 78.3 & \pm 11.5 & 79.2 & \pm 13.0 & 76.9 & \pm 8.2 & 0.509 & 76.7 & \pm 10.8 & 80.8 & \pm 12.3 & 0.220 \\
\hline Respiratory Rate, per min & 21.2 & \pm 4.4 & 22.3 & \pm 4.6 & 19.2 & \pm 3.1 & 0.012 & 21.6 & \pm 4.5 & 20.6 & \pm 4.2 & 0.397 \\
\hline \multicolumn{13}{|l|}{ Laboratory Values } \\
\hline $\mathrm{WBC}, \times 10^{9} / \mathrm{L}$ & 11.6 & \pm 8.1 & 12.2 & \pm 9.5 & 10.3 & \pm 3.6 & 0.462 & 12.5 & \pm 9.7 & 10.2 & \pm 4.3 & 0.344 \\
\hline $\mathrm{Hgb}, \mathrm{g} / \mathrm{dl}$ & 12.6 & \pm 1.8 & 12.4 & \pm 2.0 & 13.0 & \pm 1.5 & 0.322 & 12.2 & \pm 2.1 & 13.3 & \pm 1.1 & 0.065 \\
\hline Creatinine, mg/dL & 1.26 & \pm 1.1 & 1.26 & \pm 0.9 & 1.26 & \pm 1.5 & 0.981 & 1.28 & \pm 1.0 & 1.24 & \pm 1.4 & 0.913 \\
\hline Lactate, mmol/L (37 pts) & 1.48 & \pm 0.8 & 1.68 & \pm 0.8 & 0.93 & \pm 0.3 & 0.010 & 1.69 & \pm 0.9 & 1.02 & \pm 0.4 & 0.014 \\
\hline \multicolumn{13}{|l|}{ Other Characteristics } \\
\hline ED length of stay, hours & 8.63 & \pm 7.85 & 10.32 & \pm 9.13 & 5.62 & \pm 3.31 & 0.041 & 10.16 & \pm 9.23 & 6.33 & \pm 4.44 & 0.091 \\
\hline Hospital length of stay, days & N/A & N/A & 5.71 & \pm 5.94 & N/A & $\mathrm{N} / \mathrm{A}$ & N/A & 5.42 & \pm 5.10 & 7.28 & \pm 10.05 & 0.530 \\
\hline ICU, (\%) & 3 & (6) & 3 & (9) & $\mathrm{N} / \mathrm{A}$ & $\mathrm{N} / \mathrm{A}$ & N/A & 2 & (7) & 1 & (5) & 0.808 \\
\hline Mortality, (\%) & 2 & (4) & 2 & (6) & 0 & (0) & 0.279 & 2 & (7) & 0 & (0) & 0.239 \\
\hline
\end{tabular}

Data are presented as mean \pm standard deviation, or count (percentage). SBP indicates systolic blood pressure; DBP, diastolic blood pressure; WBC, white blood cell count;

ICU intensive care unit. ICU stay was recorded within current admission. Mortality was recorded up to 30 days after enrollment

significant associations between the change in $\mathrm{CO}, \mathrm{CI}$, or HR between time zero and time two hours with patient disposition from the ED (admitted vs. discharged; data not shown). However, there was a significant difference in the mean HR of patients (averaged between time zero and time two hours) who were admitted vs. discharged, 106 vs. 98 BPM, respectively $(p=0.04)$ (Fig. 1a). In patients with an infectious source, the change in $\mathrm{CO}$ and $\mathrm{CI}$ between time zero and time two hours was not statistically significant while the change in HR was $(p=$ $0.12,0.10$, and 0.03 respectively) (Fig. $1 \mathrm{~b}-\mathrm{d}$ ). SV increased significantly from time zero to time two hours, which is reflected in the fact that HR changes during this interval were significant while $\mathrm{CO}$ and $\mathrm{CI}$ changes were not (results not shown). Further, a significant difference was observed in the mean HR of patients with vs. without an infectious source (107 vs. 99 BPM; $P=0.04$ ).

An ROC analysis using initial lactate, comparing the 37 patients who had lactate measured immediately to the total cohort of 50 patients, and time zero values for $\mathrm{CO}, \mathrm{CI}$, and $\mathrm{HR}$ was used to determine the likelihood of a patient being admitted from the ED (Figure 2). The AUC $( \pm 95 \%$ Confidence Intervals) for lactate was 0.83 [0.64-0.92] compared to 0.59 [0.41-0.73] for CO, 0.68 $[0.49-0.80]$ for $\mathrm{CI}$, and $0.63[0.36-0.80]$ for HR. The ROC analysis using the difference in $\mathrm{CO}, \mathrm{CI}$, and $\mathrm{HR}$ values yielded no significant findings. When the analysis was limited to the 37 patients who had lactate, $\mathrm{CO}, \mathrm{CI}$, and HR measured initially, the AUCs for $\mathrm{CO}$ and CI decreased to $0.35 \pm 0.10$ and $0.29 \pm 0.10$ respectively.

\section{Discussion}

In this study examining a cohort of stable patients at risk for the development of sepsis after Emergency Department triage, we found that there were no significant associations between the change in $\mathrm{CO}, \mathrm{CI}$, or HR between time zero (immediately after triage) and time two hours and patient disposition from the ED (admitted or discharged to home). Similarly, there were no significant associations between the change in $\mathrm{CO}$ or $\mathrm{CI}$, between time zero (immediately after triage) and time two hours and the presence or absence of an infectious source; the change in HR from time zero to time two hours, on the other hand, was significantly different between those with an infectious and those with a non-infectious source. Further, patients admitted to the hospital vs. those discharged and those with vs. those without infectious diagnoses had higher mean heart rates during the first two hours of their ED stays. Finally, we found that the initial lactate level ordered from triage correlated much better with the likelihood of admission when compared to changes in $\mathrm{CO}, \mathrm{CI}, \mathrm{SV}$, or HR. Since both $\mathrm{CO}$ and $\mathrm{CI}$ are directly related to $\mathrm{HR}$, this suggests that in this cohort of patients the NICOM CO and CI measurements may not be better than taking a simple triage HR followed by serial HRs during the first hours of care. In 
a

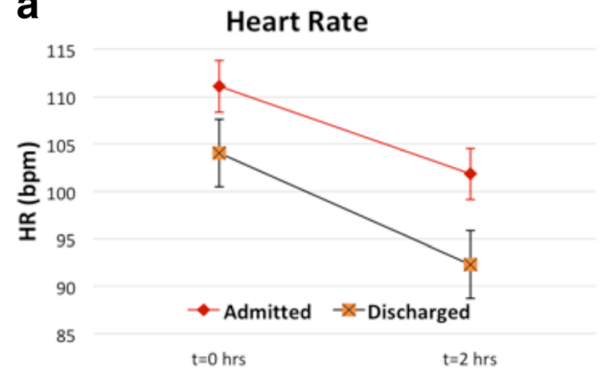

C

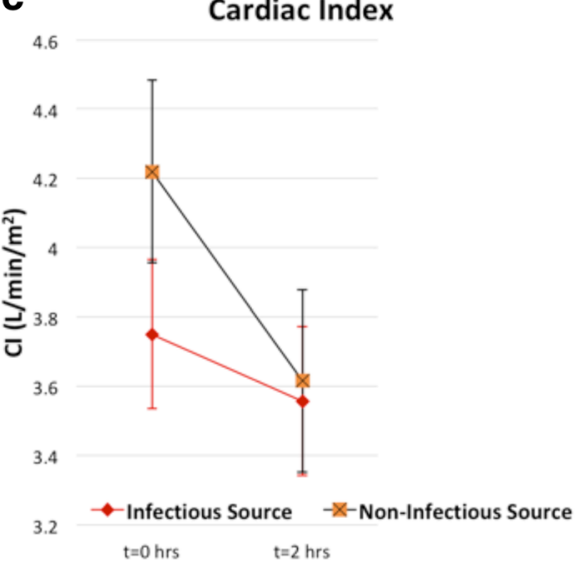

b

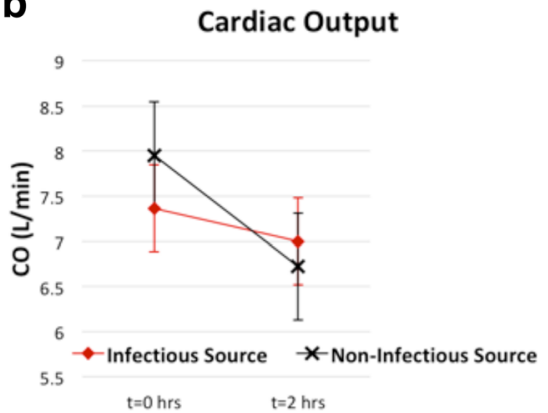

d

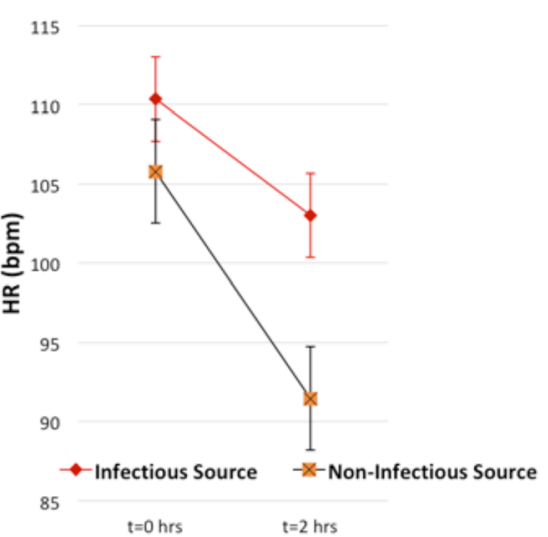

Fig. 1 Repeated measures analysis of variance. a. HR values at time zero and two hours in patients who were admitted and discharged from the $E D$. There was no significance in the association between the change in HR between $t=0$ and $t=2$ hours with patient disposition ( $F=0.62, P=$ 0.435). There was a significant difference in the mean HR when patients were grouped by disposition $(p=0.043)$. $\mathbf{b}$. CO values at time zero and two hours in patients who had an infectious source and those without an infectious source. There was a significant association between the change in $\mathrm{CO}$ between $\mathrm{t}=0$ and $\mathrm{t}=2$ hours with infection $(\mathrm{F}=2.48, P=0.122)$. There was no significant difference in the mean $\mathrm{CO}$ when patients were grouped by source $(p=0.804)$. c. Cl values at time zero and two hours in patients who had an infectious source and those without an infectious source. There was a significant association between the change in $\mathrm{Cl}$ between $\mathrm{t}=0$ and $\mathrm{t}=2$ hours with infection $(F=2.82, P=$ 0.100). There was no significant difference in the mean $\mathrm{Cl}$ when patients were grouped by source $(p=0.293)$. $\mathbf{d}$. HR values at time zero and two hours in patients who had an infectious source and those without an infectious source. There was a significant association between the change in HR between $\mathrm{t}=0$ and $\mathrm{t}=2$ hours with infection $(F=5.32, P=0.026)$. There was a significant difference in the mean HR when patients were grouped by source $(p=0.044)$. All data represented as means with error bars equal to $95 \%$ confidence interval. When associating NICOM values at time zero and two hours with another dependent variable (ie. patient disposition, LOS, or infectious source) a P-value of less than 0.25 was taken to be significant. Otherwise a $p$-value of less than 0.05 was used. CO indicates, cardiac output; Cl, cardiac index; HR, heart rate

addition, in this cohort of patients, changes in SV to preserve $\mathrm{CO}$ and $\mathrm{CI}$ did not add appreciable information to that obtained from changes observed in HR alone. Additionally, the mean HR was shown to be the value that differentiated admitted patients from discharged patients and patients with an infectious source from those with a noninfectious source. This further suggests that, in the initial ED setting, for stable patients triaged as ESI 2 and 3 , the NICOM device may not be better than a simple HR measurement to prognosticate disease severity. This study also provided evidence that lactate was a better predictor of patient disposition compared to $\mathrm{CO}, \mathrm{CI}$, and HR. Thus, given the poor predictive value of the NICOM values at triage and hour two post-triage, serum lactate obtained soon after triage remains one of the few reliable early prognosticators in stable patients at risk for development of sepsis.

Patients with a high suspicion for sepsis and obvious acute organ dysfunction at the time of triage (low blood pressure; hypoxia; mental status changes; clear signs of hypoperfusion) are generally classified as ESI 1 patients and prioritized for immediate treatment, diagnosis, and stabilization. Thus, they do not require further differentiation prior to initiation of treatment. Protocols should be created to streamline the care of these patients and dedicated resuscitation and Emergency Departmentbased Resuscitation Spaces linked to an ED-Intensive Care Unit appear to improve outcomes [22]. Less critically ill patients on triage presentation, triaged as ESI 2 or 3 , such as those recruited for this study, present 


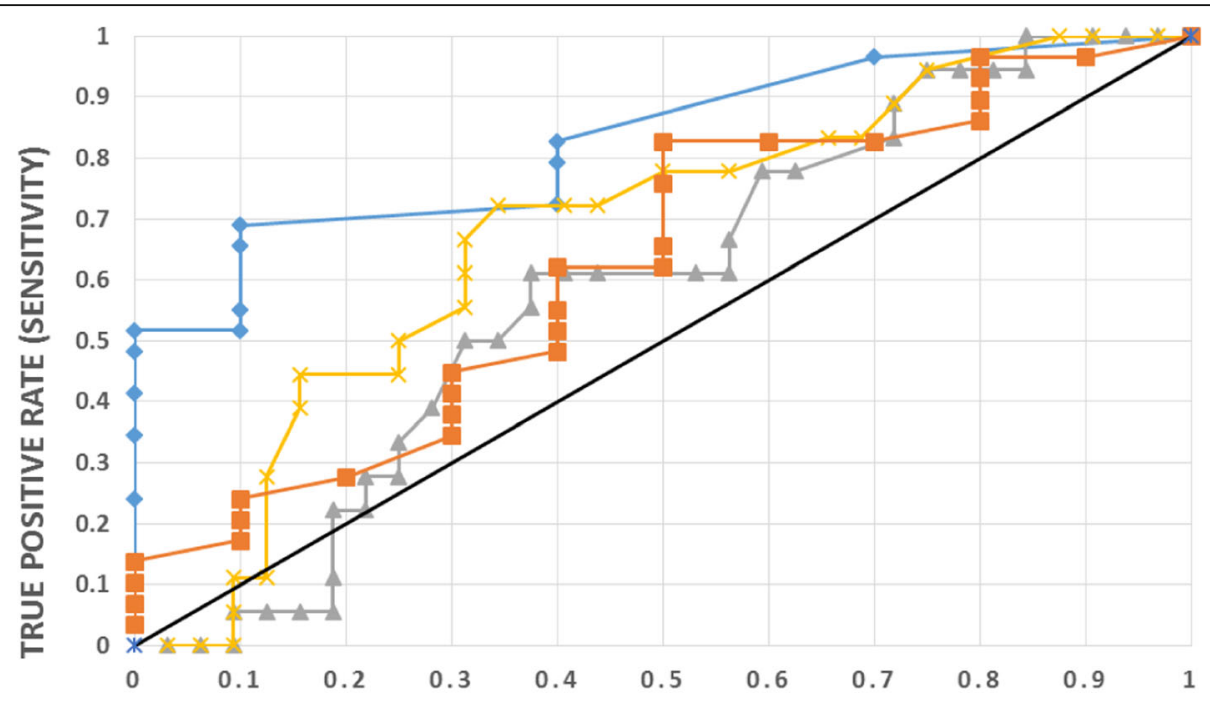

FALSE POSITIVE RATE (1 - SPECIFICITY)

$$
\rightarrow-\text { Lactate }-\mathrm{CO} \rightarrow \mathrm{Cl} \rightarrow-\mathrm{HR}
$$

Fig. 2 Receiver operator characteristic curves of lactate, $\mathrm{CO}, \mathrm{Cl}$, and $\mathrm{HR}$ at time zero. Empirical ROC curve. Area under the curve values as follow: Lactate 0.828 ; CO 0.588; Cl 0.677; HR 0.626. CO indicates, cardiac output; $\mathrm{Cl}$, cardiac index; HR, heart rate

different problems centered on rapid detection and initiation of care when compared to the more critically ill whose main issues are optimization of care. By definition, patients triaged as ESI 2 and 3 are assumed to be stable enough to wait 20 minutes to 2 hours for care. However, patients with potential sepsis are dynamic, can be rapidly changing, and when severe enough, sepsis becomes a time-sensitive disease [2327]. Patients with sepsis admitted to the hospital from the ED who are hemodynamically stable during their ED treatment have increasing mortality as initial lactate levels move from normal $(<2 \mathrm{mmol} / \mathrm{L})$ to midrange $(2-3.9 \mathrm{mmol} / \mathrm{L})$ to a level consistent with shock $(\geq 4 \mathrm{mmol} / \mathrm{L})$, with mortality increasing from 8.9 to $16 \%$ to $32.9 \%$, respectively [28]. Based on these data as well as results of other studies, early measurement of serum lactate in patients with suspected sepsis has become standard of care in sepsis protocols around the world. However, additional data to optimize risk stratification of these patients are still needed and that need provided the rationale for trialing the NICOM device to measure non-invasive hemodynamic variables at triage and two hours later. This need is highlighted by research demonstrating that validated measures of ED crowding, which is a rapidly increasing problem in United States EDs, do not impact time to fluids or antibiotics in patients presenting with obvious acute organ dysfunction but do directly impact these process measures in less sick patients admitted from the ED to the hospital with sepsis [27]. Since the mortality of this cohort of patients approached $15 \%$, it is clear that other methods of early detection are needed. In the COMMIT trial, Shapiro and colleagues randomized 64 normotensive sepsis patients with a lactate level between 2 and $4 \mathrm{mmol} / \mathrm{L}$ to volume resuscitation guided by non-invasive cardiac output monitoring vs. standard clinical care [29]. Using this strategy, they found no differences in change in SOFA score but did find that the intervention group received more intravenous fluid during the intervention period. This study differs from our study, which enrolled patients on average within 10 minutes of ED triage, in that patients were required to be enrolled within 4 hours of ED presentation and 2.5 hours of meeting eligibility criteria.

\section{Limitations}

This study should be viewed in the context of several limitations. The study population included a small convenience sample of 50 patients based on the availability of study personnel. Study personnel enrolled patients primarily during the evening hours on both weekdays and weekends. It is possible that patients who come to the ED during morning hours or overnight, meet 2 triage SIRS criteria, and have a chief complaint consistent with infection, have different risks for sepsis from the study population. It is also possible that the cohort of patients studied was not sufficiently sick from a sepsis severity perspective to show the benefit of early posttriage hemodynamic monitoring using the NICOM device since only $11 / 50$ (22\%) patients were found to 
have acute organ dysfunction (as defined by the 2nd International Sepsis Definitions conference) [30]. Specifically, we may not have included sufficient patients with early myocardial depression of sepsis to demonstrate a role for the NICOM device in risk assessment. Additionally, the authors chose to omit measuring a change in NICOM cardiac SV as a surrogate for the patient's fluid responsiveness during the passive leg raise (PLR) maneuver. A PLR is performed by changing the head of bed from a $45^{\circ}$ angle to a flat position and moving the legs and feet from a flat position to 45 degrees of elevation [21]. It was believed that a practical use of the NICOM in the triage waiting area should include measurements that can be just as quickly obtained as a set of vital signs. The initial time from study enrollment to first NICOM reading routinely took less than ten minutes. If the NICOM was incorporated into the triage procedures in a busy ED, the PLR would be cumbersome to perform and potentially delay doorto-doctor time. However, the approach taken in the study protocol may have limited early detection of potential sepsis patients by not assessing fluid responsiveness as early as possible. Patients in the study remained in the post-triage waiting room during the two hours between NICOM values and, therefore, did not receive volume resuscitation during the study period. Our samples were evenly distributed except for one outlying value in all but the HR analysis (for $\mathrm{CO}, \mathrm{CI}, \mathrm{SV}$ ) and given the sample sizes of our subgroups these outlying values may have impacted our analysis. We cannot exclude that changes in NICOM values between time zero and time 2 hours may reflect "noise" emanating from the measuring device. However, very clear trends in changes in NICOM values were seen: decreasing heart rate, increasing stroke volume, and slight decreases in cardiac output and cardiac index. Further, it is unlikely that these observations reflect a "white coat phenomenon." $\mathrm{Pa}-$ tients were seen prior to enrollment by a triage nurse, not a physician however the white coat phenomenon can occur with contact with any category of health care provider. More likely, the exertion of walking into the hospital and the anxiety about their medical condition contributed to the initial elevation in heart rate noted at triage and on time zero NICOM readings. Lying still and decreased stress will lead to decreased heart rate and this is reflected in the second NICOM readings. In addition, our patients did not receive fluid boluses between time zero and time twohour NICOM measurements and this may have contributed to our lack of detection of NICOM as a tool for risk stratification. In fact, the NICOM device may be most helpful for fluid management as a study by Oord and colleagues demonstrated that a $500 \mathrm{ml}$ fluid bolus did not reveal SV changes while a $1000 \mathrm{ml}$ bolus was sufficient to demonstrate hemodynamic responses [31]. Finally, questions have been raised about the sensitivity and specificity of easily implemented, non-invasive methods to assess fluid responsiveness such as the NICOM device when compared to left ventricular outflow tract echocardiography, which is considered the non-invasive gold standard correlating most accurately with the values obtained from PAC [32].

\section{Future directions}

Future work informed by this study should incorporate PLR performed immediately after the time zero NICOM values and the change in SV from time zero and two hours into the data. If patients have a positive response to PLR demonstrating fluid responsiveness, then they should have immediate treatment, including fluid bolus, initiated. Studies comparing NICOM CO measurements in a baseline semi-recumbent position versus changes observed when the PLR is performed and in hemodynamic measurements in patients undergoing the PLR versus those getting a fluid bolus have shown conflicting results [33-36]. Generalization of some of these results is limited by the study populations being limited almost exclusively to patients in intensive care units. Other vital signs and their trends including blood pressure, shock index (HR/SBP), and RR have been shown to be important in risk stratification of undifferentiated patients in the ED [37-39] and novel machine learning techniques integrating these values with lactate, other inflammatory and perfusion markers, along with hemodynamic measurements may yield better results in future studies.

\section{Conclusions}

In conclusion, this study found that NICOM CO and CI, obtained at two separate time points after ED triage, do not help with early differentiation of patients at risk for sepsis who do not have obvious organ dysfunction at triage. This lack of differentiation occurred when assessed in relation to their disposition from the ED or the presence or absence of infection. While some significance was observed when comparing changes in $\mathrm{CO}, \mathrm{CI}, \mathrm{SV}$, and $\mathrm{HR}$ to the presence of infection, this association was driven primarily by the significant differences in the mean HR of patients who have an infectious source. NICOM appears to have a role in the hemodynamic monitoring of sepsis patients during proximal resuscitation but the results of our study do not support its use as a risk stratification tool. Furthermore, even though the study found a significant difference in the mean HR of patients who were admitted versus 
those who were discharged, a serum lactate still served as a better predictor of patient admission from the ED.

\section{Appendix}

Appendix: 50 Chief Complaints from the Advanced Triage Protocol

1. Abdominal Distention

2. Abscess

3. Chills

4. Cough

5. Chest Pain

6. Cerebral Vascular Accident/Transient Ischemic Attack

7. Dehydrated

8. Diarrhea

9. Diff Breathing

10. Dizzy

11. Dyspnea

12. Fatigue

13. Fever

14. Genitourinary

15. Headache

16. Hematuria

17. Hemoptysis

18. Hyperglycemia

19. Hypoglycemia

20. Infection

21. Infection, Extremity

22. Infection, Eye

23. Infection, Face

24. Infection, Foot

25. Infection, Hand

26. Infection, Joint

27. Infection, Leg

28. Infection, Sinus

29. Infection, Wound

30. Inflammation

31. Jaundice

32. Lethargy

33. Lightheadedness

34. Low Blood Pressure

35. Mental Status Change

36. Nausea/Vomiting

37. Nausea/Vomiting/Diarrhea

38. Neutropenia

39. Pain, Abdomen

40. Pain, Chest

41. Pain, Flank

42. Rash

43. Shortness of Breath

44. Sore Throat

45. Swell

46. Upper Respiratory Signs
47. Urinary Tract Infection

48. Vomiting

49. Weak

50. Wound Check

\section{Acknowledgements}

The authors would like to acknowledge the kindness and inquisitiveness of the patients who consented to participate in this study as research cannot be performed without willing research subjects who, often, will not directly benefit from the research being conducted.

Data for this study can be obtained by contacting DFG @

david.gaieski@jefferson.edu and the dataset will be forwarded in a

reasonable time frame to the requesting person.

\section{Authors' contributions}

SBC: study concept and design, acquisition of the data, analysis and interpretation of the data, drafting of the manuscript. DFG: study concept and design, acquisition of the data, analysis and interpretation of the data, drafting of the manuscript, critical revision of the manuscript for important intellectual content, and acquisition of equipment for trial. AB: analysis and interpretation of the data, critical revision of the manuscript for important intellectual content. LMG: study concept and design, acquisition of the data, analysis and interpretation of the data, drafting of the manuscript, critical revision of the manuscript for important intellectual content. FSS: analysis and interpretation of the data, statistical expertise, drafting of the manuscript. AJD: study concept and design, analysis and interpretation of the data, drafting of the manuscript. The author(s) read and approved the final manuscript.

\section{Funding}

No funding was obtained for this study; equipment was provided by Cheetah Medical, which had no role in the design of the study, analysis of the data, or writing and review of the manuscript.

Ethics approval and consent to participate

All participants provided informed consent to participate in this study.

\section{Consent for publication}

Consent for publication of data was obtained and the study was approved by the institutional review board.

\section{Competing interests}

None of the authors has any relevant conflict of interest to disclose related to this work.

\section{Author details \\ 'Department of Emergency Medicine, Advocate Sherman Hospital, Elgin, IL, USA. ${ }^{2}$ Department of Emergency Medicine, Sidney Kimmel Medical College at Thomas Jefferson University, 1025 Walnut Street; 300 College Building, 19107 Philadelphia, PA, USA. ${ }^{3}$ Department of Internal Medicine, Division of Pulmonary and Critical Care Medicine, Cleveland Clinic, Cleveland, OH, USA. ${ }^{4}$ Department of Obstetrics and Gynecology, Hospital of the University of Pennsylvania, Philadelphia, PA, USA. ${ }^{5}$ Department of Emergency Medicine, Perelman School of Medicine, University of Pennsylvania, Philadelphia, PA, USA.}

Received: 8 February 2020 Accepted: 12 January 2021

Published online: 28 January 2021

\section{References}

1. Gaieski DF, Edwards JM, Kallan MJ, Carr BG. Benchmarking the Incidence and Mortality of Severe Sepsis in the United States. Crit Care Med. 2013; 41(5):1167-74.

2. Singer M, Deutschman CS, Seymour CW, ShankarHari M, Annane D, Bauer $M$, et al. The Third International Consensus Definitions for Sepsis and Septic Shock (Sepsis-3). JAMA. 2016;315(8):801-10.

3. Ahrens T. Hemodynamics in Sepsis. AACN Advanced Critical Care. 2006; 17(4):435-45. 
4. Rivers EP, Nguyen B, Havstad S, Ressler J, Muzzin A, Knoblich B, et al. Early Goal-Directed Therapy in the Treatment of Severe Sepsis and Septic Shock. N Engl J Med. 2001;345(19):1368-77.

5. Faix JD. Biomarkers of Sepsis. Crit Rev Clin Lab Sci. 2013;50(1):23-36.

6. Bloos F, Reinhart K. Rapid diagnosis of sepsis. Virulence. 2014;5(1):154-60.

7. Wacker C, Prkno A, Brunkhorst FM, Schlattmann P. Procalcitonin as a diagnostic marker for sepsis: a systematic review and meta-analysis. Lancet Infect Dis. 2013;13(5):426-35.

8. Thomas-rueddel DO, Poidinger B, Weiss M, Bach F, Dey K, Häberle H, et al. Hyperlactatemia is an independent predictor of mortality and denotes distinct subtypes of severe sepsis and septic shock. J Crit Care. 2015;30(2): 439.e1-6.

9. Casserly B, Phillips GS, Schorr C, Dellinger P, Townsend S, Osborn T, et al. Lactate measurements in sepsis-induced tissue hypoperfusion: results from the surviving sepsis campaign database. Crit Care Med. 2015;43(3):567-73.

10. Velissaris D, Karmouzos V, Kotroni I, Pierrakos C, Karanikolas M. The Use of Pulmonary Artery Catheter in Sepsis Patients: A Literature Review. J Clin Med Res. 2016;8(11):769-76.

11. De Backer D. Ultrasonic evaluation of the heart. Curr Opin Crit Care. 2014; 20(3):309-14.

12. Parmley $\mathrm{CL}$, Pousman RM. Noninvasive cardiac output monitoring. Curr Opin Anaesthesiol. 2002;15(6):675-80.

13. Napoli AM, Machan JT, Corl K, Forcada A. The use of impedance cardiography in predicting mortality in emergency department patients with severe sepsis and septic shock. Acad Emerg Med. 2010;17(4):452-5.

14. Marik PE. Noninvasive Cardiac Output Monitors: A State-of the-Art Review. J Cardiothorac Vasc Anesth. 2013;27(1):121-34

15. Squara P, Denjean D, Estagnasie P, Brusset A, Dib JC, Dubois C. Noninvasive cardiac output monitoring (NICOM): a clinical validation. Intensive Care Med. 2007;33(7):1191-4

16. Raval NY, Squara P, Cleman M, Yalamanchili K, Winklmaier M, Burkhoff D. Multicenter evaluation of noninvasive cardiac output measurement by bioreactance technique. J Clin Monit Comput. 2008;22(2):113-9.

17. Lin TW, Luo CF, Lin CC, Lee WC, Su BC. Utilization of bioreactance technique as indicator for preload responsiveness during living donor liver donation. Transplant Proc. 2014:46(3):669-71.

18. Waldron NH, Miller TE, Thacker JK, Manchester AK, White WD, Nardiello J, et al. A prospective comparison of a noninvasive cardiac output monitor versus esophageal Doppler monitor for goal-directed fluid therapy in colorectal surgery patients. Anesth Analg. 2014;118(5):966-75.

19. Dunham CM, Chirichella TJ, Gruber BS, Ferrari JP, Martin JA, Luchs BA, et al. Emergency department noninvasive (NICOM) cardiac outputs are associated with trauma activation, patient injury severity and host conditions and mortality. J Trauma Acute Care Surg. 2012;73(2):479-85.

20. Dunham CM, Chirichella TJ, Gruber BS, Ferrari JP, Martin JA, Luchs BA, et al. In emergently ventilated trauma patients, low end-tidal CO2 and low cardiac output are associated and correlate with hemodynamic instability, hemorrhage, abnormal pupils, and death. BMC Anesthesiol. 2013;13(1):20

21. Duus N, Shogilev DJ, Skibsted S, Zijlstra HW, Fish E, Oren-Grinberg A, et al. The reliability and validity of passive leg raise and fluid bolus to assess fluid responsiveness in spontaneously breathing emergency department patients. J Crit Care. 2015;30(1):217.e1-5.

22. Gunnerson KJ, Bassin BS, Havey RA, Haas NL, Sozener CB, Medlin RP, et al. Association of an Emergency Department-Based Intensive Care Unit With Survival and Inpatient Intensive Care Unit Admissions. JAMA Network Open. 2019;2(7):e197684.

23. The ProCESS Investigators. A randomized trial of protocol-based care for early septic shock. N Engl J Med. 2014;370:1683-93.

24. The ARISE Investigators and the ANZICS Clinical Trials Group. Goal-directed resuscitation for patients with early septic shock. N Engl J Med. 2014;371: 1496-506.

25. Mouncey PR, Osborn TM, Power S, Harrison DA, Zia Sadique M, Grieve RD, et al. Trial of Early, Goal-Directed Resuscitation for Septic Shock. N Engl J Med. 2015;372:1301-11.

26. Seymour CW, Gesten F, Prescott HC, Friedrich ME, Iwashyna TJ, Phillips GS, et al. Time to Treatment and Mortality during Mandated Emergency Care for Sepsis. N Engl J Med. 2017;376:2235-44.

27. Gaieski DF, Agarwal AK, Mikkelsen ME, Drumheller B, Sante CS, Shofer FS, et al. The Impact of ED Crowding on Early Interventions and Mortality in Patients with Severe Sepsis. Am J Emerg Med. 2017;35(7):953-60.
28. Levy MM, Fink MP, Marshall JC, Abraham E, Angus D, Cook D, et al. 200 SCCM/ESICM/ACCP/ATS/SIS International Sepsis Definitions Conference. Crit Care Med. 2003;31(4):1250-6.

29. Hou PC, Filbin MR, Napoli A, Feldman J, Pang PS, Sankoff J, et al. Cardiac Output Monitoring Managing Intravenous Therapy (COMMIT) to Treat Emergency Department Patients with Sepsis. Shock. 2016;46(2):132-6.

30. Levy MM, Fink MP, Marshall JC, Abraham E, Angus D, Cook D, et al., for the International Sepsis Definitions Conference. 2001 SCCM/ESICM/ACCP/ATS/ SIS International Sepsis Definitions Conference. Intensive Care Med. 2003;29: 530-538.

31. Oord M, Olgers TJ, Doff-Holman M, Harms MP, Ligtenberg JJ, Ter Maaten JC. Ultrasound and NICOM in the assessment of fluid responsiveness in patients with mild sepsis in the emergency department: a pilot study. BMJ Open. 2017;7(1):e013465. Published 2017 Jan 27.

32. McGregor D, Sharma S, Gupta S, Ahmed S, Harris T. Emergency department non-invasive cardiac output study (EDNICO): an accuracy study. Scandanarian Journal of Trauma, Resuscitation and Emergency Medicine. 2020;28(8).

33. Mikkelsen ME, Miltiades AN, Gaieski DF, Goyal M, Fuchs BD, Shah CV, et al. Serum lactate is associated with mortality in severe sepsis independent of organ failure and shock. Crit Care Med. 2009;37(5):1670-7.

34. Shapiro $\mathrm{NI}$, Howell MD, Talmor D, Nathanson LA, Lisbon A, Wolfe RE, et al. Serum lactate as a predictor of mortality in emergency department patients with infection. Ann Emerg Med. 2005;45(5):524-8.

35. Benomar B, Ouattara A, Estagnasie P, Brusset A, Squara P. Fluid responsiveness predicted by noninvasive bioreactance-based passive leg raise test. Intensive Care Med. 2010;36(11):1875-81.

36. Elwan MH, Roshdy A, Reynolds JA, Elsharkawy EM, Eltahan SM, Coats TJ. What is the normal haemodynamic response to passive leg raise? A study of healthy volunteers. Emerg Med J. 2018;35:529-9.

37. Quinten VM, van Meurs M, Ter Maaten JC, Ligtenberg JJ. Trends in vital signs and routine biomarkers in patients with sepsis during resuscitation in the emergency department: a prospective observational pilot study. BMJ Open. 2016;6(5):e009718

38. Quinten VM, van Meurs M, Olgers TJ, Vonk JM, Ligtenberg JJM, Ter Maaten JC. Repeated vital sign measurements in the emergency department predict patient deterioration within 72 hours: a prospective observational study. Scand J Trauma Resusc Emerg Med. 2018;26(1):57.

39. Heather Farley MT, Zubrow J, Gies P, Kolm S, Mascioli DD, Mahoney, William $\mathrm{S}$, Weintraub. Emergency department tachypnea predicts transfer to a higher level of care in the first 24 hours after ED admission. Acad Emerg Med. 2010;17(7):718-22.

\section{Publisher's Note}

Springer Nature remains neutral with regard to jurisdictional claims in published maps and institutional affiliations.
Ready to submit your research? Choose BMC and benefit from:

- fast, convenient online submission

- thorough peer review by experienced researchers in your field

- rapid publication on acceptance

- support for research data, including large and complex data types

- gold Open Access which fosters wider collaboration and increased citations

- maximum visibility for your research: over $100 \mathrm{M}$ website views per year

At $\mathrm{BMC}$, research is always in progress.

Learn more biomedcentral.com/submissions 